\title{
Strong convergence theorems for three-steps iterations for asymptotically nonexpansive mappings in Banach spaces
}

\section{Cui Yun An', Zuo Zhan Fei and Henryk Hudzik ${ }^{3 *}$}

\section{"Correspondence:}

hudzik@amu.edu.pl

${ }^{3}$ Faculty of Mathematics and

Computer Science, Adam

Mickiewicz University,

Umultowska 87, 61-614, Poznan,

Poland

Full list of author information is

available at the end of the article

\begin{abstract}
We consider the problem of the convergence of the three-steps iterative sequences for asymptotically nonexpansive mappings in a real Banach space. Under suitable conditions, it has been proved that the iterative sequence converges strongly to a fixed point, which is also a solution of certain variational inequality. The results presented in this paper extend and improve some recent results.
\end{abstract}

Keywords: asymptotically nonexpansive mapping; three-steps iteration; fixed points; uniformly Gâteaux differentiable norm

\section{Introduction}

Let $X$ be a real Banach space with dual $X^{*}, J: X \rightarrow 2^{X^{*}}$ denotes the normalized duality mapping from $X$ into $X^{*}$ given by

$$
J(x)=\left\{f \in X^{*}:\langle x, f\rangle=\|x\|\|f\| \wedge\|f\|=\|x\|\right\}, \quad \forall x \in X .
$$

Let $C$ be a subset of $X$. A mapping $T: C \rightarrow C$ is called contraction if there exists a constant $\alpha \in(0,1)$ such that $\|T x-T y\| \leq \alpha\|x-y\|$ for any $x, y \in C$. The mapping $T$ is called nonexpansive if $\|T x-T y\| \leq\|x-y\|$ for any $x, y \in C$, and it is called asymptotically nonexpansive if there exists a sequence $\left\{k_{n}\right\}$ in the interval $[1, \infty)$ with $\lim _{n \rightarrow \infty} k_{n}=1$ and such that

$$
\left\|T^{n} x-T^{n} y\right\| \leq k_{n}\|x-y\|
$$

for all $x, y \in C$ and all $n \in N$, where $N$ is the set of natural numbers.

The class of asymptotically nonexpansive mappings was introduced by Goebel and Kirk [1] as an important generalization of nonexpansive mappings. They proved that if $C$ is a nonempty, bounded, closed and convex subset of a real uniformly convex Banach space and $T$ is an asymptotically nonexpansive self-mapping of $C$, then $T$ has a fixed point in $C$. In 2000, Noor [2] introduced a three-steps iterative scheme and studied the approximate solutions of a variational inclusion in Hilbert spaces. In 2002, Xu and Noor [3] introduced and studied a new class of three-steps iterative schemes for solving the nonlinear equation $T x=x$ for asymptotically nonexpansive mappings $T$ in uniformly convex Banach spaces. In 2006, Nilsrakoo and Saejung [4] defined a three-steps mean value iterative scheme and

C) 2013 An et al: licensee Springer. This is an Open Access article distributed under the terms of the Creative Commons Attribution License (http://creativecommons.org/licenses/by/2.0), which permits unrestricted use, distribution, and reproduction in any medium, provided the original work is properly cited. 
extended the results of $\mathrm{Xu}$ and Noor [3]. In 2007, Yao and Noor [5] made a refinement and improvement of the previously known results.

Now we define a new three-steps iteration scheme for asymptotically nonexpansive mappings as follows.

Definition 1.1 Let $X$ be a Banach space, $C$ be a nonempty convex subset of $X, T: C \rightarrow C$ be an asymptotically nonexpansive mapping and $f: C \rightarrow C$ be a contraction. For a given $x_{1} \in C$ and $n \in N$, let us define the sequences $\left\{x_{n}\right\},\left\{y_{n}\right\}$ and $\left\{z_{n}\right\}$ by the iterative scheme

$$
\left\{\begin{array}{l}
z_{n}=\left(1-\gamma_{n}\right) x_{n}+\gamma_{n} T^{n} x_{n}, \\
y_{n}=\left(1-\beta_{n}\right) x_{n}+\beta_{n} T^{n} z_{n}, \\
x_{n+1}=\left(1-\alpha_{n}\right) f\left(x_{n}\right)+\alpha_{n} T^{n} y_{n},
\end{array}\right.
$$

where $\left\{\alpha_{n}\right\},\left\{\beta_{n}\right\}$ and $\left\{\gamma_{n}\right\}$ are approximate sequences in $[0,1]$.

If $\gamma_{n} \equiv 0$, then the iterations defined in (2) reduces to the two-steps iterations defined as follows.

Definition 1.2 For a given $x_{1} \in C$ and $n \geq 1$, let us define the sequences $\left\{x_{n}\right\}$ and $\left\{y_{n}\right\}$ by the iterative scheme

$$
\left\{\begin{array}{l}
y_{n}=\left(1-\beta_{n}\right) x_{n}+\beta_{n} T^{n} x_{n}, \\
x_{n+1}=\left(1-\alpha_{n}\right) f\left(x_{n}\right)+\alpha_{n} T^{n} y_{n},
\end{array}\right.
$$

where $\left\{\alpha_{n}\right\}$ and $\left\{\beta_{n}\right\}$ are approximate sequences in $[0,1]$.

If $\beta_{n}=\gamma_{n} \equiv 0$, then the iterations defined in (2) reduces to the one-step iterations defined as follows.

Definition 1.3 For a given $x_{1} \in C$ and $n \in \mathbb{N}$, define the sequence $\left\{x_{n}\right\}$ by the iterative scheme

$$
x_{n+1}=\left(1-\alpha_{n}\right) f\left(x_{n}\right)+\alpha_{n} T^{n} x_{n},
$$

where $\left\{\alpha_{n}\right\}$ is an approximate sequence in $[0,1]$.

The purpose of this paper is to establish a strong convergence theorem of the three-steps iterations for asymptotically nonexpansive mappings in a real Banach space equipped with a uniformly Gâteaux differentiable norm and to present some corollaries. Our results extend and improve the corresponding ones announced by Ceng et al. [6], Chang et al. [7], Lou et al. [8], Shahzad and Udomene [9] and others.

\section{Preliminaries}

Throughout this paper, we will use the following notions. Let $X$ be a real Banach space with the norm $\|\cdot\|$ and let $X^{*}$ be its dual space. When $\left\{x_{n}\right\}$ is a sequence in $X$, then $x_{n} \rightarrow x$ (respectively $x_{n} \rightarrow x, x_{n} \rightarrow x$ ) will denote the strong (respectively the weak, the weak star) 
convergence of the sequence $\left\{x_{n}\right\}$ to $x$. Of course, the weak star convergence is considered in Banach spaces $X$ which are dual spaces. We shall denote the single-valued duality mapping and the set of fixed points for a mapping $T$ by $j$ and $F(T)$, respectively.

Definition 2.1 Let $S_{X}$ denote the unit sphere of a Banach space $X$. The space $X$ is said to have a uniformly Gâteaux differentiable norm $\|\cdot\|$, if for each $y \in C$ the limit

$$
\lim _{t \rightarrow 0} \frac{\|x+t y\|-\|x\|}{t}
$$

exists uniformly with respect to $x \in S_{X}$.

It is well known [10] that if $X$ is equipped with a uniformly Gâteaux differentiable norm, then any duality mapping on $X$ is single-valued and it is norm-to-weak" uniformly continuous, that is, $x_{n} \rightarrow x$ implies that $j\left(x_{n}\right) \rightarrow j(x)$.

Lemma 2.2 [11] Let $X$ be a real Banach space. Then for each $x, y \in X$, the following inequality holds:

$$
\|x+y\|^{2} \leq\|x\|^{2}+2(y, j(x+y)), \quad \forall j(x+y) \in J(x+y) .
$$

Lemma 2.3 [12] Let $\left\{a_{n}\right\},\left\{b_{n}\right\},\left\{c_{n}\right\}$ be three nonnegative real sequences satisfying

$$
a_{n+1} \leq\left(1-t_{n}\right) a_{n}+b_{n}+c_{n}
$$

with $\left\{t_{n}\right\} \subset[0,1], \sum_{n=0}^{\infty} t_{n}=\infty, b_{n}=o\left(t_{n}\right)$, and $\sum_{n=0}^{\infty} c_{n}<\infty$. Then $a_{n} \rightarrow 0$.

Now, we start with our first result.

Lemma 2.4 Let $X$ be a real Banach space, $C$ be a nonempty convex subset of $X$ and $T: C \rightarrow$ $C$ be an asymptotically nonexpansive mapping defined by (1) with $F(T) \neq \emptyset$ and $\left\{x_{n}\right\}_{n=1}^{\infty}$ be the composite process defined by iterative scheme (2). Then the sequence $\left\{x_{n}\right\}$ is bounded.

Proof Let $p \in F(T)$ and $\gamma^{\prime}=\sup _{n}\left\{3\left(k_{n}\right)^{3}: n \geq 1\right\}$. We have from (2) that

$$
\begin{aligned}
\left\|z_{n}-p\right\| \leq & \left(1-\gamma_{n}\right)\left\|x_{n}-p\right\|+\gamma_{n}\left\|T^{n} x_{n}-p\right\| \leq\left(1+\gamma_{n}\left(k_{n}-1\right)\right)\left\|x_{n}-p\right\|, \\
\left\|y_{n}-p\right\| \leq & \left(1-\beta_{n}\right)\left\|x_{n}-p\right\|+\beta_{n}\left\|T^{n} z_{n}-p\right\| \leq\left(1-\beta_{n}\right)\left\|x_{n}-p\right\| \\
& +\beta_{n} k_{n}\left\|z_{n}-p\right\| \leq\left(1+\beta_{n}\left(k_{n}-1\right)\left(1+\gamma_{n} k_{n}\right)\right)\left\|x_{n}-p\right\|,
\end{aligned}
$$

and so

$$
\begin{aligned}
\left\|x_{n+1}-p\right\| & \leq\left(1-\alpha_{n}\right)\left\|f\left(x_{n}\right)-p\right\|+\alpha_{n}\left\|T^{n} y_{n}-p\right\| \\
& \leq \alpha\left(1-\alpha_{n}\right)\left\|x_{n}-p\right\|+\left(1-\alpha_{n}\right)\|f(p)-p\|+\alpha_{n} k_{n}\left\|y_{n}-p\right\| \\
& \leq\left(\alpha\left(1-\alpha_{n}\right)+\alpha_{n} k_{n}\left(1+\beta_{n}\left(k_{n}-1\right)\left(1+\gamma_{n} k_{n}\right)\right)\right)\left\|x_{n}-p\right\|+\left(1-\alpha_{n}\right)\|f(p)-p\| \\
& \leq\left(\alpha-\alpha \alpha_{n}+\alpha_{n} k_{n}+\alpha_{n} \beta_{n} k_{n}^{2}+\alpha_{n} \beta_{n} k_{n}^{3}-\alpha_{n} \beta_{n} k_{n}-\alpha_{n} \beta_{n} \gamma_{n} k_{n}^{2}\right)\left\|x_{n}-p\right\|
\end{aligned}
$$




$$
\begin{aligned}
& +\left(1-\alpha_{n}\right)\|f(p)-p\| \\
\leq & \left(\alpha+\alpha_{n}\left(3 k_{n}^{3}-\alpha\right)\right)\left\|x_{n}-p\right\|+\left(1-\alpha_{n}\right)\|f(p)-p\| \\
\leq & \left(\alpha+\alpha_{n}\left(\gamma^{\prime}-\alpha\right)\right)\left\|x_{n}-p\right\|+\left(1-\alpha_{n}\right)\|f(p)-p\| \\
= & \frac{\left(\alpha+\alpha_{n}\left(\gamma^{\prime}-\alpha\right)\right)}{\gamma^{\prime}} \gamma^{\prime}\left\|x_{n}-p\right\|+\frac{\left(1-\alpha_{n}\right)\left(\gamma^{\prime}-\alpha\right)}{\gamma^{\prime}} \frac{\gamma^{\prime}\|f(p)-p\|}{\gamma^{\prime}-\alpha} .
\end{aligned}
$$

Hence, it follows by induction that

$$
\left\|x_{n}-p\right\| \leq \max \left\{\gamma^{\prime}\left\|x_{0}-p\right\|, \frac{\gamma^{\prime}}{\gamma^{\prime}-\alpha}\|f(p)-p\|\right\} .
$$

Therefore, $\left\{x_{n}\right\}$ is bounded.

In order to prove our results, we also need the following lemma; see [8].

Lemma 2.5 Let $X$ be a real Banach space equipped with a uniformly Gâteaux differentiable norm, $C$ a bounded, closed and convex subset of $X, T: C \rightarrow C$ an asymptotically nonexpansive mapping defined by (1) with $F(T) \neq \emptyset, f: C \rightarrow C$ a contraction with the contraction constant $\alpha$. For any $\left\{\alpha_{n}\right\} \subset(0,1)$ define the sequence of contractions $T_{n}^{f}: C \rightarrow C$ by $T_{n}^{f}(\tilde{z})=\left(1-\alpha_{n}\right) f(\tilde{z})+\alpha_{n} T^{n} \tilde{z}$, where $\alpha_{n}<\frac{1-\alpha}{k_{n}-\alpha}, \lim _{n \rightarrow \infty} \alpha_{n}=1$ and $\lim _{n \rightarrow \infty} \frac{k_{n}-1}{1-\alpha_{n}}=0$. Let $\tilde{z}_{n} \in C$ be the unique fixed points of $T_{n}^{f}$, that is,

$$
\tilde{z}_{n}=\left(1-\alpha_{n}\right) f\left(\tilde{z}_{n}\right)+\alpha_{n} T^{n} \tilde{z}_{n}, \quad \forall n \in N .
$$

Then the sequence $\left\{\tilde{z}_{n}\right\}$ converges strongly to the unique solution of the following variational solution $p$ :

$$
p \in F(T) \text { and }\left\langle(I-f) p, J\left(p-x^{*}\right)\right\rangle \leq 0, \quad \forall x^{*} \in F(T) .
$$

Lemma 2.6 Let $C$ be a closed convex subset of a real Banach space $X, T: C \rightarrow C$ be an asymptotically nonexpansive mapping and $f: C \rightarrow C$ be a contraction with the contraction constant $\alpha$. Let us assume that there are given three sequences $\left\{\alpha_{n}\right\},\left\{\beta_{n}\right\}$ and $\left\{\gamma_{n}\right\}$ in $[0,1]$ satisfying the following conditions:

(i) $\alpha_{n}<\frac{1-\alpha}{k_{n}-\alpha}, \lim _{n \rightarrow \infty} \alpha_{n}=1, \sum_{n=0}^{\infty}\left(1-\alpha_{n}\right)=\infty$ and $\lim _{n \rightarrow \infty} \frac{k_{n}-1}{1-\alpha_{n}}=0$;

(ii) $\sum_{n=1}^{\infty}\left(\left|\alpha_{n}-\alpha_{n-1}\right|+\left|\beta_{n}-\beta_{n-1}\right|+\left|\gamma_{n}-\gamma_{n-1}\right|\right)<\infty$ and that $\left\{x_{n}\right\}_{n=1}^{\infty}$ is the composite process defined by the iterative scheme (2). Then we have the following assertions:

(a) $\lim _{n \rightarrow \infty}\left\|x_{n+1}-x_{n}\right\|=0$;

(b) if $\lim \sup _{n} \beta_{n}\left(1+\gamma_{n}\right)<1$, then $\lim _{n \rightarrow \infty}\left\|x_{n}-T x_{n}\right\|=0$.

Proof (a) By Lemma 2.4, we know that the sequence $\left\{x_{n}\right\}$ is bounded. Hence, it follows that the sequences $\left\{f\left(x_{n}\right)\right\},\left\{y_{n}\right\},\left\{T^{n} x_{n}\right\}$ and $\left\{T^{n} z_{n}\right\}$ are also bounded. Therefore, we have from (2) that

$$
\begin{aligned}
\left\|z_{n}-z_{n-1}\right\|= & \|\left(1-\gamma_{n}\right)\left(x_{n}-x_{n-1}\right)+\gamma_{n}\left(T^{n} x_{n}-T^{n} x_{n-1}\right) \\
& +\gamma_{n}\left(T^{n} x_{n-1}-T^{n-1} x_{n-1}\right)+\left(\gamma_{n-1}-\gamma_{n}\right)\left(x_{n-1}-T^{n-1} x_{n-1}\right) \|
\end{aligned}
$$




$$
\begin{aligned}
\leq & \left(1-\gamma_{n}\right)\left\|x_{n}-x_{n-1}\right\|+\gamma_{n} k_{n}\left\|x_{n}-x_{n-1}\right\| \\
& +\gamma_{n}\left\|T^{n} x_{n-1}-T^{n-1} x_{n-1}\right\|+\left|\gamma_{n}-\gamma_{n-1}\right|\left\|x_{n-1}-T^{n-1} x_{n-1}\right\| \\
= & \left(1+\gamma_{n}\left(k_{n}-1\right)\right)\left\|x_{n}-x_{n-1}\right\|+\gamma_{n}\left\|T^{n} x_{n-1}-T^{n-1} x_{n-1}\right\| \\
& +\left|\gamma_{n}-\gamma_{n-1}\right|\left\|x_{n-1}-T^{n-1} x_{n-1}\right\|
\end{aligned}
$$

and

$$
\begin{aligned}
\left\|y_{n}-y_{n-1}\right\|= & \left\|\left(1-\beta_{n}\right) x_{n}+\beta_{n} T^{n} z_{n}-\left(1-\beta_{n-1}\right) x_{n-1}-\beta_{n-1} T^{n-1} z_{n-1}\right\| \\
= & \|\left(1-\beta_{n}\right)\left(x_{n}-x_{n-1}\right)+\beta_{n}\left(T^{n} z_{n}-T^{n} z_{n-1}\right) \\
& +\beta_{n}\left(T^{n} z_{n-1}-T^{n-1} z_{n-1}\right)+\left(\beta_{n-1}-\beta_{n}\right)\left(x_{n-1}-T^{n-1} z_{n-1}\right) \| \\
\leq & \left(1-\beta_{n}\right)\left\|x_{n}-x_{n-1}\right\|+\beta_{n} k_{n}\left\|z_{n}-z_{n-1}\right\| \\
& +\beta_{n}\left\|T^{n} z_{n-1}-T^{n-1} z_{n-1}\right\|+\left|\beta_{n}-\beta_{n-1}\right|\left\|x_{n-1}-T^{n-1} z_{n-1}\right\| \\
\leq & \left(1-\beta_{n}\right)\left\|x_{n}-x_{n-1}\right\|+\beta_{n} k_{n}\left(1+\gamma_{n}\left(k_{n}-1\right)\right)\left\|x_{n}-x_{n-1}\right\| \\
& +k_{n} \beta_{n} \gamma_{n}\left\|T^{n} x_{n-1}-T^{n-1} x_{n-1}\right\|+\beta_{n}\left\|T^{n} z_{n-1}-T^{n-1} z_{n-1}\right\| \\
& +k_{n} \beta_{n}\left|\gamma_{n}-\gamma_{n-1}\right|\left\|x_{n-1}-T^{n-1} x_{n-1}\right\| \\
& +\left|\beta_{n}-\beta_{n-1}\right|\left\|x_{n-1}-T^{n-1} z_{n-1}\right\|,
\end{aligned}
$$

where it follows that

$$
\begin{aligned}
\left\|x_{n+1}-x_{n}\right\|= & \|\left(1-\alpha_{n}\right)\left(f\left(x_{n}\right)-f\left(x_{n-1}\right)\right)+\alpha_{n}\left(T^{n} y_{n-1}-T^{n-1} y_{n-1}\right) \\
& +\left(\alpha_{n-1}-\alpha_{n}\right)\left(f\left(x_{n-1}\right)-T^{n-1} y_{n-1}\right)+\alpha_{n}\left(T^{n} y_{n}-T^{n} y_{n-1}\right) \| \\
\leq & \alpha\left(1-\alpha_{n}\right)\left\|x_{n}-x_{n-1}\right\|+\alpha_{n}\left\|T^{n} y_{n-1}-T^{n-1} y_{n-1}\right\| \\
& +\left|\alpha_{n}-\alpha_{n-1}\right|\left\|f\left(x_{n-1}\right)-T^{n-1} y_{n-1}\right\|+\alpha_{n} k_{n}\left\|y_{n}-y_{n-1}\right\| \\
\leq & \left(\alpha+\alpha_{n}(1-\alpha)\right)\left\|x_{n}-x_{n-1}\right\| \\
& +\alpha_{n}\left(k_{n}-1\right)\left(1+\beta_{n} k_{n}\left(1+\gamma_{n} k_{n}\right)\right)\left\|x_{n}-x_{n-1}\right\| \\
& +\alpha_{n}\left\|T^{n} y_{n-1}-T^{n-1} y_{n-1}\right\|+\alpha_{n} \beta_{n} k_{n}\left\|T^{n} z_{n-1}-T^{n-1} z_{n-1}\right\| \\
& +\alpha_{n} \beta_{n} \gamma_{n} k_{n}^{2}\left\|T^{n} x_{n-1}-T^{n-1} x_{n-1}\right\| \\
& +\left|\alpha_{n}-\alpha_{n-1}\right|\left\|f\left(x_{n-1}\right)-T^{n-1} y_{n-1}\right\| \\
& +\alpha_{n} k_{n}\left|\beta_{n}-\beta_{n-1}\right|\left\|x_{n-1}-T^{n-1} z_{n-1}\right\| \\
& +\alpha_{n} \beta_{n} k_{n}^{2}\left|\gamma_{n}-\gamma_{n-1}\right|\left\|x_{n-1}-T^{n-1} x_{n-1}\right\| \\
\leq & \left(1-(1-\alpha)\left(1-\alpha_{n}\right)\right)\left\|x_{n}-x_{n-1}\right\| \\
& +\alpha_{n}\left(k_{n}-1\right)\left(1+\beta_{n} k_{n}\left(1+\gamma_{n} k_{n}\right)\right)\left\|x_{n}-x_{n-1}\right\| \\
& +\alpha_{n}\left\|T^{n} y_{n-1}-T^{n-1} y_{n-1}\right\|+\alpha_{n} k_{n}\left\|T^{n} z_{n-1}-T^{n-1} z_{n-1}\right\| \\
& +\alpha_{n} k_{n}^{2}\left\|T^{n} x_{n-1}-T^{n-1} x_{n-1}\right\|+\left|\alpha_{n}-\alpha_{n-1}\right|\left\|f\left(x_{n-1}\right)-T^{n-1} y_{n-1}\right\| \\
& +\alpha_{n} k_{n}\left|\beta_{n}-\beta_{n-1}\right|\left\|x_{n-1}-T^{n-1} z_{n-1}\right\| \\
& (1) \\
& \\
&
\end{aligned}
$$




$$
\begin{aligned}
& +\alpha_{n} \beta_{n} k_{n}^{2}\left|\gamma_{n}-\gamma_{n-1}\right|\left\|x_{n-1}-T^{n-1} x_{n-1}\right\| \\
\leq & \left(1-(1-\alpha)\left(1-\alpha_{n}\right)\right)\left\|x_{n}-x_{n-1}\right\| \\
& +\alpha_{n}\left(k_{n}-1\right)\left(1+\beta_{n} k_{n}\left(1+\gamma_{n} k_{n}\right)\right)\left\|x_{n}-x_{n-1}\right\| \\
& +\alpha_{n}\left(k_{n}^{12}-1\right) M+M\left(\left|\alpha_{n}-\alpha_{n-1}\right|+\left|\beta_{n}-\beta_{n-1}\right|+\left|\gamma_{n}-\gamma_{n-1}\right|\right) \\
\leq & \left(1-(1-\alpha)\left(1-\alpha_{n}\right)\right)\left\|x_{n}-x_{n-1}\right\|+(1-\alpha)\left(1-\alpha_{n}\right) \frac{\alpha_{n}\left(k_{n}-1\right)}{(1-\alpha)\left(1-\alpha_{n}\right)} \\
& \times\left[\left(1+\beta_{n} k_{n}\left(1+\gamma_{n} k_{n}\right)\right)+\left(k_{n}^{6}+1\right)\left(k_{n}^{3}+1\right)\left(k_{n}^{2}+k_{n}+1\right)\right] M \\
& +M\left(\left|\alpha_{n}-\alpha_{n-1}\right|+\left|\beta_{n}-\beta_{n-1}\right|+\left|\gamma_{n}-\gamma_{n-1}\right|\right),
\end{aligned}
$$

where

$$
\begin{aligned}
M= & \max \left\{\left\|x_{n}-x_{n-1}\right\|,\left\|T^{n} y_{n-1}-T^{n-1} y_{n-1}\right\|,\left\|f\left(x_{n-1}\right)-T^{n-1} y_{n-1}\right\|,\right. \\
& k_{n}\left(\left\|x_{n-1}-T^{n-1} z_{n-1}\right\|,\left\|T^{n} z_{n-1}-T^{n-1} z_{n-1}\right\|\right), \\
& \left.k_{n}^{2}\left(\left\|x_{n-1}-T^{n-1} x_{n-1}\right\|,\left\|T^{n} x_{n-1}-T^{n-1} x_{n-1}\right\|\right)\right\} .
\end{aligned}
$$

Obviously, by condition (i), we have $t_{n}=(1-\alpha)\left(1-\alpha_{n}\right) \rightarrow 0$ and

$$
\begin{aligned}
\frac{b_{n}}{t_{n}} & =\frac{\alpha_{n}\left(k_{n}-1\right)}{(1-\alpha)\left(1-\alpha_{n}\right)}\left[\left(1+\beta_{n} k_{n}\left(1+\gamma_{n} k_{n}\right)\right)+\left(k_{n}^{6}+1\right)\left(k_{n}^{3}+1\right)\left(k_{n}^{2}+k_{n}+1\right)\right] M \\
& \rightarrow 0 \quad(n \rightarrow \infty) .
\end{aligned}
$$

It follows from Lemma 2.3 and condition (ii) that $\lim _{n \rightarrow \infty}\left\|x_{n+1}-x_{n}\right\|=0$.

(b) By (a), $\lim _{n \rightarrow \infty}\left\|x_{n+1}-x_{n}\right\|=0$, so it follows from (2) and (i) that $\left\|x_{n+1}-T^{n} y_{n}\right\|=$ $\left(1-\alpha_{n}\right)\left\|f\left(x_{n}\right)-T^{n} y_{n}\right\| \rightarrow 0$ and

$$
\left\|x_{n}-T^{n} y_{n}\right\| \leq\left\|x_{n+1}-x_{n}\right\|+\left\|x_{n+1}-T^{n} y_{n}\right\| \rightarrow 0 .
$$

Now, we will prove that $\lim _{n \rightarrow \infty}\left\|x_{n}-T^{n} x_{n}\right\|=0$. We have from (2) that

$$
\begin{aligned}
\left\|x_{n}-T^{n} x_{n}\right\| & \leq\left\|x_{n}-T^{n} y_{n}\right\|+\left\|T^{n} y_{n}-T^{n} x_{n}\right\| \\
& \leq\left\|x_{n}-T^{n} y_{n}\right\|+k_{n}\left\|x_{n}-y_{n}\right\| \\
& =\left\|x_{n}-T^{n} y_{n}\right\|+\beta_{n} k_{n}\left\|x_{n}-T^{n} z_{n}\right\| \\
& \leq\left\|x_{n}-T^{n} y_{n}\right\|+\beta_{n} k_{n}\left\|x_{n}-T^{n} x_{n}\right\|+\beta_{n} k_{n}\left\|T^{n} x_{n}-T^{n} z_{n}\right\| \\
& \leq\left\|x_{n}-T^{n} y_{n}\right\|+\beta_{n} k_{n}\left\|x_{n}-T^{n} x_{n}\right\|+\beta_{n} k_{n}^{2}\left\|x_{n}-z_{n}\right\| \\
& =\left\|x_{n}-T^{n} y_{n}\right\|+\beta_{n} k_{n}\left(1+\gamma_{n} k_{n}\right)\left\|x_{n}-T^{n} x_{n}\right\| .
\end{aligned}
$$

It follows from (7) that $\lim _{n \rightarrow \infty}\left\|x_{n}-T^{n} x_{n}\right\| \leq \beta_{n}\left(1+\gamma_{n}\right) \lim _{n \rightarrow \infty}\left\|x_{n}-T^{n} x_{n}\right\|$, where, by the condition $\lim \sup _{n} \beta_{n}\left(1+\gamma_{n}\right)<1$, we have that

$$
\lim _{n \rightarrow \infty}\left\|x_{n}-T^{n} x_{n}\right\|=0
$$


Finally, we will show that $\lim _{n \rightarrow \infty}\left\|x_{n}-T x_{n}\right\|=0$. In fact, according to (8), we have

$$
\begin{aligned}
\left\|x_{n+1}-T^{n} x_{n+1}\right\| & \leq\left\|x_{n+1}-x_{n}\right\|+\left\|x_{n}-T^{n} x_{n}\right\|+\left\|T^{n} x_{n}-T^{n} x_{n+1}\right\| \\
& \leq\left(1+k_{n}\right)\left\|x_{n+1}-x_{n}\right\|+\left\|x_{n}-T^{n} x_{n}\right\| \rightarrow 0 .
\end{aligned}
$$

Hence,

$$
\begin{aligned}
\left\|x_{n+1}-T x_{n+1}\right\| & \leq\left\|x_{n+1}-T^{n+1} x_{n+1}\right\|+\left\|T^{n+1} x_{n+1}-T x_{n+1}\right\| \\
& \leq\left\|x_{n+1}-T^{n+1} x_{n+1}\right\|+k_{1}\left\|T^{n} x_{n+1}-x_{n+1}\right\| \rightarrow 0,
\end{aligned}
$$

which implies that $\lim _{n \rightarrow \infty}\left\|x_{n}-T x_{n}\right\|=0$.

\section{Main results}

Theorem 3.1 Let $X$ be a real Banach space equipped with a uniformly Gâteaux differentiable norm, $C$ be a bounded, closed and convex subset of $X, T: C \rightarrow C$ be an asymptotically nonexpansive mapping defined by (1) with $F(T) \neq \emptyset$ and $f: C \rightarrow C$ be a contraction with the contraction constant $\alpha$. Let $\left\{x_{n}\right\}$ be the sequence defined by the iterative scheme (2) with $\left\{\alpha_{n}\right\},\left\{\beta_{n}\right\}$ and $\left\{\gamma_{n}\right\}$ satisfying the following conditions:

(C $\left.C_{1}\right) \alpha_{n}<\frac{1-\alpha}{k_{n}-\alpha}, \lim _{n \rightarrow \infty} \alpha_{n}=1, \sum_{n=0}^{\infty}\left(1-\alpha_{n}\right)=\infty$ and $\lim _{n \rightarrow \infty} \frac{k_{n}-1}{1-\alpha_{n}}=0$;

$\left(\mathrm{C}_{2}\right) \limsup _{n} \beta_{n}\left(1+\gamma_{n}\right)<1$;

$\left(\mathrm{C}_{3}\right) \sum_{n=1}^{\infty}\left(\left|\alpha_{n}-\alpha_{n-1}\right|+\left|\beta_{n}-\beta_{n-1}\right|+\left|\gamma_{n}-\gamma_{n-1}\right|\right)<\infty$.

Then the sequence $\left\{x_{n}\right\}$ converges strongly to the unique solution $p$ of the variational inequality:

$$
p \in F(T) \quad \text { and } \quad\left\langle(I-f) p, J\left(p-x^{*}\right)\right\rangle \leq 0, \quad \forall x^{*} \in F(T)
$$

Proof Since $C$ is closed, by Lemma 2.4, $\left\{x_{n}\right\}$ is bounded, so $\left\{f\left(x_{n}\right)\right\},\left\{y_{n}\right\},\left\{T^{n} x_{n}\right\}$ and $\left\{T^{n} z_{n}\right\}$ are also bounded. Let $\left\{\tilde{z}_{n}\right\}$ be the sequence defined by

$$
\tilde{z}_{n}=\left(1-\alpha_{n}\right) f\left(\tilde{z}_{n}\right)+\alpha_{n} T^{n} \tilde{z}_{n}, \quad \forall n \in N
$$

It follows from Lemma 2.5 that the sequence $\left\{\tilde{z}_{n}\right\}$ converges strongly to a fixed point $p$ of $T$ and $p$ is also the unique solution of the variational inequality (6). We will next prove that

$$
\limsup _{n \rightarrow \infty}\left\langle f(p)-p, j\left(x_{n}-p\right)\right\rangle \leq 0
$$

By Lemma 2.6(b), $\lim _{n \rightarrow \infty}\left\|x_{n}-T x_{n}\right\|=0$. It is easy to show that

$$
\left\|T^{m} x_{n}-x_{n}\right\| \rightarrow 0 \quad(n \rightarrow \infty), \forall m \in N
$$

where if we put $P_{n}(m)=\left\|T^{m} x_{n}-x_{n}\right\|\left(2 k_{m}\left\|\tilde{z}_{m}-x_{n}\right\|+\left\|T^{m} \tilde{z}_{n}-x_{n}\right\|\right)$, then $P_{n}(m) \rightarrow 0$ as $n \rightarrow \infty$. On the other hand, we have from (10) that

$$
\tilde{z}_{m}-x_{n}=\left(1-\alpha_{m}\right)\left(f\left(\tilde{z}_{m}\right)-x_{n}\right)+\alpha_{m}\left(T^{m} \tilde{z}_{m}-x_{n}\right) .
$$


It follows by Lemma 2.2 that

$$
\begin{aligned}
\left\|\tilde{z}_{m}-x_{n}\right\|^{2} \leq & \alpha_{m}^{2}\left\|T^{m} \tilde{z}_{m}-x_{n}\right\|^{2}+2\left(1-\alpha_{m}\right)\left\langle f\left(\tilde{z}_{m}\right)-x_{n}, j\left(\tilde{z}_{m}-x_{n}\right)\right\rangle \\
\leq & \alpha_{m}^{2}\left(\left\|T^{m} \tilde{z}_{m}-T^{m} x_{n}\right\|+\left\|T^{m} x_{n}-x_{n}\right\|\right)^{2} \\
& +2\left(1-\alpha_{m}\right)\left(\left\langle f\left(\tilde{z}_{m}\right)-\tilde{z}_{m}, j\left(\tilde{z}_{m}-x_{n}\right)\right\rangle+\left\|\tilde{z}_{m}-x_{n}\right\|^{2}\right) \\
\leq & \alpha_{m}^{2}\left(k_{m}\left\|\tilde{z}_{m}-x_{n}\right\|+\left\|T^{m} x_{n}-x_{n}\right\|\right)^{2} \\
& +2\left(1-\alpha_{m}\right)\left(\left\langle f\left(\tilde{z}_{m}\right)-\tilde{z}_{m}, j\left(\tilde{z}_{m}-x_{n}\right)\right\rangle+k_{m}^{2}\left\|\tilde{z}_{m}-x_{n}\right\|^{2}\right) \\
= & \alpha_{m}^{2} k_{m}^{2}\left\|\tilde{z}_{m}-x_{n}\right\|^{2}+\alpha_{m}^{2} P_{n}(m)+2\left(1-\alpha_{m}\right) k_{m}^{2}\left\|\tilde{z}_{m}-x_{n}\right\|^{2} \\
& +2\left(1-\alpha_{m}\right)\left\langle f\left(\tilde{z}_{m}\right)-\tilde{z}_{m}, j\left(\tilde{z}_{m}-x_{n}\right)\right\rangle \\
= & k_{m}^{2}\left(1+\left(1-\alpha_{m}\right)^{2}\right)\left\|\tilde{z}_{m}-x_{n}\right\|^{2}+\alpha_{m}^{2} P_{n}(m) \\
& +2\left(1-\alpha_{m}\right)\left\langle f\left(\tilde{z}_{m}\right)-\tilde{z}_{m}, j\left(\tilde{z}_{m}-x_{n}\right)\right\rangle .
\end{aligned}
$$

Hence,

$$
\left\langle\tilde{z}_{m}-f\left(\tilde{z}_{m}\right), j\left(\tilde{z}_{m}-x_{n}\right)\right\rangle \leq \frac{k_{m}^{2}-1+k_{m}^{2}\left(1-\alpha_{m}\right)^{2}}{2\left(1-\alpha_{m}\right)}\left\|\tilde{z}_{m}-x_{n}\right\|^{2}+\frac{\alpha_{m}^{2} P_{n}(m)}{2\left(1-\alpha_{m}\right)}
$$

Since $\lim _{n \rightarrow \infty}\left\|x_{n}-T x_{n}\right\|=0$ and the sequences $\left\{\tilde{z}_{n}\right\}$ and $\left\{x_{n}\right\}$ are bounded it follows that for some constant $M>\sup _{m, n}\left\|\tilde{z}_{m}-x_{n}\right\|$, we have

$$
\limsup _{n \rightarrow \infty}\left\langle\tilde{z}_{m}-f\left(\tilde{z}_{m}\right), j\left(\tilde{z}_{m}-x_{n}\right)\right\rangle \leq \frac{M}{2}\left(\left(k_{m}+1\right) \frac{k_{m}-1}{1-\alpha_{m}}+2 k_{m}^{2}\left(1-\alpha_{m}\right)\right) .
$$

Since $\tilde{z}_{m} \rightarrow p \in F(T)$ as $m \rightarrow \infty$ and the duality mapping is norm-to-weakly* uniformly continuous, we obtain that

$$
\limsup _{n \rightarrow \infty}\left\langle f(p)-p, j\left(x_{n}-p\right)\right\rangle \leq 0
$$

Finally, we will show that $x_{n} \rightarrow p$. We have

$$
\begin{aligned}
\left\|z_{n}-p\right\| & \leq\left(1-\gamma_{n}\right)\left\|x_{n}-p\right\|+\gamma_{n}\left\|T^{n} x_{n}-p\right\| \leq k_{n}\left\|x_{n}-p\right\|, \\
\left\|y_{n}-p\right\| & \leq\left(1-\beta_{n}\right)\left\|x_{n}-p\right\|+\beta_{n}\left\|T^{n} z_{n}-p\right\| \\
& \leq\left(1-\beta_{n}\right)\left\|x_{n}-p\right\|+\beta_{n} k_{n}\left\|z_{n}-p\right\| \\
& \leq\left(1-\beta_{n}+\beta_{n} k_{n}^{2}\right)\left\|x_{n}-p\right\| \leq k_{n}^{2}\left\|x_{n}-p\right\|
\end{aligned}
$$

and so

$$
\begin{aligned}
\left\|x_{n+1}-p\right\|^{2}= & \left\|\left(1-\alpha_{n}\right)\left(f\left(x_{n}\right)-p\right)+\alpha_{n}\left(T^{n} y_{n}-p\right)\right\|^{2} \\
\leq & \alpha_{n}^{2} k_{n}^{2}\left\|y_{n}-p\right\|^{2}+2\left(1-\alpha_{n}\right)\left\langle f\left(x_{n}\right)-p, J\left(x_{n+1}-p\right)\right\rangle \\
\leq & \alpha_{n}^{2} k_{n}^{6}\left\|x_{n}-p\right\|^{2}+\alpha\left(1-\alpha_{n}\right)\left(\left\|x_{n}-p\right\|+\left\|x_{n+1}-p\right\|\right)^{2} \\
& +2\left(1-\alpha_{n}\right)\left\langle f(p)-p, J\left(x_{n+1}-p\right)\right\rangle
\end{aligned}
$$




$$
\begin{aligned}
\leq & \left(\alpha\left(1-\alpha_{n}\right)+\alpha_{n}^{2}\right)\left\|x_{n}-p\right\|^{2}+\alpha\left(1-\alpha_{n}\right)\left\|x_{n+1}-p\right\|^{2} \\
& \alpha_{n}^{2}\left(k_{n}^{6}-1\right)\left\|x_{n}-p\right\|^{2}+2\left(1-\alpha_{n}\right)\left(f(p)-p, J\left(x_{n+1}-p\right)\right\rangle \\
\leq & \left(1-(2-\alpha)\left(1-\alpha_{n}\right)\right)\left\|x_{n}-p\right\|^{2}+\alpha\left(1-\alpha_{n}\right)\left\|x_{n+1}-p\right\|^{2} \\
& +\left(\left(1-\alpha_{n}\right)^{2}+\left(k_{n}-1\right)\right) M+2\left(1-\alpha_{n}\right) \tilde{\gamma}_{n+1},
\end{aligned}
$$

where $\tilde{\gamma}_{n+1}=\max \left\{\left\langle f(p)-p, J\left(x_{n+1}-p\right)\right\rangle, 0\right\}$. By (11), we have $\tilde{\gamma}_{n+1} \rightarrow 0$, and $M>\sup _{n}\left(k_{n}^{5}+\right.$ $\left.k_{n}^{4}+k_{n}^{3}+k_{n}^{2}+k_{n}+1\right)\left\|x_{n}-p\right\|$. Then it follows that

$$
\begin{aligned}
\left\|x_{n+1}-p\right\|^{2} \leq & \frac{1-(2-\alpha)\left(1-\alpha_{n}\right)}{1-\alpha\left(1-\alpha_{n}\right)}\left\|x_{n}-p\right\|^{2}+\frac{2\left(1-\alpha_{n}\right)}{1-\alpha\left(1-\alpha_{n}\right)} \tilde{\gamma}_{n+1} \\
& +\left(\frac{\left(1-\alpha_{n}\right)^{2}}{1-\alpha\left(1-\alpha_{n}\right)}+\frac{\left(k_{n}-1\right)}{1-\alpha\left(1-\alpha_{n}\right)}\right) M .
\end{aligned}
$$

If we define $a_{n}=\left\|x_{n}-p\right\|^{2}, t_{n}=\frac{2(1-\alpha)\left(1-\alpha_{n}\right)}{1-\alpha\left(1-\alpha_{n}\right)}$ and $b_{n}=\frac{\left(1-\alpha_{n}\right)^{2}+\left(k_{n}-1\right)}{1-\alpha\left(1-\alpha_{n}\right)} M$, then applying Lemma 2.3 we conclude that $x_{n} \rightarrow p$. Moreover, it follows from (12) that $p$ satisfies condition (9). In order to show that $p$ is unique, let $p^{*} \in F$ be another solution of (9) in $F$. Then adding the inequalities $\left\langle f(p)-p, j\left(p^{*}-p\right)\right\rangle \leq 0$ and $\left\langle f\left(p^{*}\right)-p^{*}, j\left(p-p^{*}\right)\right\rangle \leq 0$, we get that $(1-\alpha)\left\|p-p^{*}\right\|^{2} \leq 0$, which implies the equality $p=p^{*}$.

The following example gives a mapping $T$, which is not nonexpansive but satisfying all the assumptions of Theorem 3.1.

Example 3.2 Let $B$ denote the unit ball in the Hilbert space $l^{2}$ and let $T: B \rightarrow B$ be defined as follows:

$$
T:\left(x_{1}, x_{2}, x_{3}, \ldots\right) \rightarrow\left(0,2 x_{1}, A_{2} x_{2}, A_{3} x_{3}, \ldots\right)
$$

where $A_{n}=\left(1-\frac{1}{n}\right)^{2}\left(\frac{2}{n+1}-\frac{1}{n(n+2)}\right), n=2,3, \ldots$. Then it is easy to verify that $T$ is an asymptotically nonexpansive mapping with $k_{n}=2 \prod_{i=2}^{n} A_{i}=1+\frac{1}{n^{2}}$, but it is not nonexpansive. If we set $\alpha_{n}=1-\frac{1}{n}, f(x)=\frac{1}{2} x$ and $\beta_{n}=\gamma_{n}=\frac{1}{2}$, then the real sequences $\left\{\alpha_{n}\right\},\left\{\beta_{n}\right\}$ and $\left\{\gamma_{n}\right\}$ satisfy conditions $\left(C_{1}\right),\left(C_{2}\right)$ and $\left(C_{3}\right)$ from Theorem 3.1 , and it is easy to prove that 0 is the unique fixed point of $T$ in $B$.

If $\gamma_{n} \equiv 0$ in Theorem 3.1, then we have by (2) that $z_{n}=x_{n}$. In fact, we have the following corollary.

Corollary 3.3 Let X be a real Banach space equipped with a uniformly Gâteaux differentiable norm, $C$ be a bounded closed convex subset of $X, T: C \rightarrow C$ be an asymptotically nonexpansive mapping defined by (1) with $F(T) \neq \emptyset$ and $f: C \rightarrow C$ be a contraction with the contraction constant $\alpha$. Let $\left\{x_{n}\right\}$ be the sequence defined by the iterative scheme (3) with $\left\{\alpha_{n}\right\}$ and $\left\{\beta_{n}\right\}$ satisfying the following conditions:

$\left(\mathrm{C}_{1}\right) \alpha_{n}<\frac{1-\alpha}{k_{n}-\alpha}, \lim _{n \rightarrow \infty} \alpha_{n}=1, \sum_{n=0}^{\infty}\left(1-\alpha_{n}\right)=\infty$ and $\lim _{n \rightarrow \infty} \frac{k_{n}-1}{1-\alpha_{n}}=0$;

$\left(\mathrm{C}_{2}\right) \lim \sup _{n} \beta_{n}<1$;

$\left(\mathrm{C}_{3}\right) \sum_{n=1}^{\infty}\left(\left|\alpha_{n}-\alpha_{n-1}\right|+\left|\beta_{n}-\beta_{n-1}\right|\right)<\infty$. 
Then the sequence $\left\{x_{n}\right\}$ converges strongly to the unique solution $p$ of the variational inequality (9).

If $\beta_{n}=\gamma_{n} \equiv 0$ in Theorem 3.1, then we have by (2) that $z_{n}=y_{n}=x_{n}$. Hence, it follows that the following result is satisfied.

Corollary 3.4 Let $X$ be a real Banach space equipped with a uniformly Gâteaux differentiable norm, $C$ be a bounded closed convex subset of $X, T: C \rightarrow C$ be an asymptotically nonexpansive mapping defined by (1) with $F(T) \neq \emptyset$ and $f: C \rightarrow C$ be a contraction with the contraction constant $\alpha$. Let $\left\{x_{n}\right\}$ be the sequence defined by the iterative scheme (4) with $\left\{\alpha_{n}\right\}$ satisfying the following conditions:

(C) $\alpha_{n}<\frac{1-\alpha}{k_{n}-\alpha}, \lim _{n \rightarrow \infty} \alpha_{n}=1$ and $\lim _{n \rightarrow \infty} \frac{k_{n}-1}{1-\alpha_{n}}=0$;

$\left(\mathrm{C}_{2}\right) \sum_{n=0}^{\infty}\left(1-\alpha_{n}\right)=\infty$;

$\left(C_{3}\right)$ either $\sum_{n=1}^{\infty}\left|\alpha_{n}-\alpha_{n-1}\right|<\infty$ or $\lim _{n \rightarrow \infty}\left(\alpha_{n+1} / \alpha_{n}\right)=1$.

Then the sequence $\left\{x_{n}\right\}$ converges strongly to the unique solution of the variational inequality (9).

\section{Remarks 3.5}

1. If $\gamma_{n} \equiv 0$ and $f=u$ is a constant function in Theorem 3.1, then the iterative scheme (2) reduces to the following iterative scheme:

$$
\left\{\begin{array}{l}
y_{n}=\left(1-\beta_{n}\right) x_{n}+\beta_{n} T^{n} x_{n}, \\
x_{n+1}=\left(1-\alpha_{n}\right) u+\alpha_{n} T^{n} y_{n} .
\end{array}\right.
$$

In consequence, Corollary 3.3 improves Theorem 1 of Chang et al. from [7].

2. Let in Theorem $3.1 \gamma_{n} \equiv 0$ and the iterative scheme (3) be replaced by the following scheme:

$$
\left\{\begin{array}{l}
y_{n}=\left(1-\beta_{n}\right) x_{n}+\beta_{n} T^{n} x_{n}, \\
x_{n+1}=\left(1-\alpha_{n}\right) f\left(x_{n}\right)+\alpha_{n} y_{n} .
\end{array}\right.
$$

Then by Theorem 3.1, we have the more general result than the result of Lou et al. from [8] and Corollary 3.6 of Ceng et al. in [6]. If $T$ and $\left\{\alpha_{n}\right\}$ are as in Corollary 3.4, assume that $\left\{\beta_{n}\right\},\left\{\gamma_{n}\right\} \in[0,1], \alpha_{n}+\beta_{n}+\gamma_{n}=1$ and $0<\liminf _{n} \beta_{n} \leq \lim \sup _{n} \beta_{n}<1$. Then the sequence $\left\{x_{n}\right\}$ defined by $x_{n+1}=\alpha_{n} f\left(x_{n}\right)+\beta_{n} x_{n}+\gamma_{n} T^{n} x_{n}$ converges strongly to the unique solution of the variational inequality (9).

3. Theorem 3.1 and Corollary 3.4 extend Theorem 3.3 of Shahzad and Udomene in [9] to a more wide class of spaces. 


\section{Author details}

${ }^{1}$ Department of Mathematics, Harbin University of Science and Technology, Harbin, 150080, P.R. China. ${ }^{2}$ Department of Mathematics and Statistics, Chongqing Three Gorges University, Wanzhou, 404000, P.R. China. ${ }^{3}$ Faculty of Mathematics and Computer Science, Adam Mickiewicz University, Umultowska 87, 61-614, Poznan, Poland.

\section{Acknowledgements}

The first author is supported by foundation of Heilongjiang Province education department (12521070). The second author is supported by the fund of Scientific research in Southeast University (the support project of fundamental research). The last author is supported by the State Committee for Scientific Research, Poland, Grant No. N N201 362236

\section{Received: 26 September 2012 Accepted: 28 March 2013 Published: 7 June 2013}

\section{References}

1. Goebel, K, Kirk, WA: A fixed point theorem for asymptotically nonexpansive mappings. Proc. Am. Math. Soc. 35, 171-174 (1972)

2. Noor, MA: New approximation schemes for general variational inequalities. J. Math. Anal. Appl. 251, 217-229 (2000)

3. Xu, BL, Noor, MA: Fixed point iterations for asymptotically nonexpansive mappings in Banach spaces. J. Math. Anal. Appl. 267, 444-453 (2002)

4. Nilsrakoo, W, Saejung, S: A new three-steps fixed point iteration scheme for asymptotically nonexpansive mappings. Appl. Math. Comput. 181, 1026-1034 (2006)

5. Yao, Y, Noor, MA: Convergence of three-steps iterations for asymptotically nonexpansive mappings. Appl. Math. Comput. 187, 883-892 (2007)

6. Ceng, LC, Xu, HK, Yao, JC: The viscosity approximation method for asymptotically nonexpansive mappings in Banach spaces. Nonlinear Anal. 69(4), 1402-1412 (2008)

7. Chang, SS, Joseph Lee, HW, Chan, CK: On Reich's strong convergence theorem for asymptotically nonexpansive mappings in Banach spaces. Nonlinear Anal. 66, 2364-2374 (2007)

8. Lou, J, Zhang, L-j, He, Z: Viscosity approximation methods for asymptotically nonexpansive mappings. Appl. Math. Comput. 203(1), 171-177 (2008)

9. Shahzad, N, Udomene, A: Fixed point solutions of variational inequalities for asymptotically nonexpansive mappings in Banach spaces. Nonlinear Anal. 64, 558-567 (2006)

10. Takahashi, W, Ueda, Y: On Reich's strong convergence theorems for resolvents of accretive operators. J. Math. Anal. Appl. 104, 546-553 (1984)

11. Morales, $\mathrm{CH}$, Jung, JS: Convergence of paths for pseudo-contractive mappings in Banach spaces. Proc. Am. Math Soc. 128, 3411-3419 (2000)

12. Liu, LS: Ishikawa and Mann iterafive processes with errors for nonlinear strongly accretive mappings in Banach spaces. J. Math. Anal. Appl. 194, 114-125 (1995)

\section{Submit your manuscript to a SpringerOpen ${ }^{\ominus}$ journal and benefit from:}

- Convenient online submission

- Rigorous peer review

- Immediate publication on acceptance

Open access: articles freely available online

- High visibility within the field

- Retaining the copyright to your article 